\title{
Early Evaluation of Root System Architecture in Maize Using X-ray Microscopy of Root Tips
}

\author{
Keith Duncan, Ni Jiang, Tyler Dowd and Christopher Topp \\ Donald Danforth Plant Science Center, Saint Louis, Missouri, United States
}

Root systems are the critical organs that provide all water and nutrients to the plant, but how they occupy the soil in three dimensions is poorly understood. Root system architecture (RSA) is a difficult phenomenon to evaluate in meaningful detail in situ, and a wide range of proxy strategies have been developed to measure RSA and the traits that contribute to it, both in the lab and the field [1,2]. Successful measurement of any trait that contributes to understanding RSA involves prediction of how roots will behave in the field under a range of environmental pressures. Progress has been made in that direction using gel-based optical projection tomography to measure root growth at early stages that successfully predicted RSA development in mature field-grown plants of the same variety [3].

We have demonstrated that X-ray microscopy (XRM) has utility in generating data-rich 3D scans of plant organs, tissues, and cells, providing morphological image volumes that allow visualization and measurement of complex structures. We are using XRM to study roots at the cellular level to determine how micro-structure drives macroscale patterning, using two maize lines that have known differences in RSA at later stages when grown under identical environmental conditions. Conventional electron microscopy sample preparation methods have been successfully adapted for imaging plant samples with XRM [4-7]. We have optimized these methods for improved contrast using a range of fixation, post-fix, and contrast agents, that has yielded volume data that is easier to analyze computationally (Fig 1). We are also using a microwave tissue processor to enhance the efficacy of fixation and contrast solutions, and to reduce sample processing steps from days down to hours.

These improvements in sample preparation and imaging allowed us to develop computational protocols, using both manual and machine learning models, that can segment and measure tissues and individual cells from XRM scan data (Fig 2). Successful and accurate segmentation, particularly of cells in the root elongation zone, allow direct comparison of cell size, shape, volume, and distribution in 3D space between the two maize lines, and yield superior data than was possible using extrapolation from $2 \mathrm{D}$ sections. This strategy will ultimately allow us to identify the genetic factor(s) that contribute to differences in RSA and can be used in breeding programs to improve maize performance in the field [7]. 

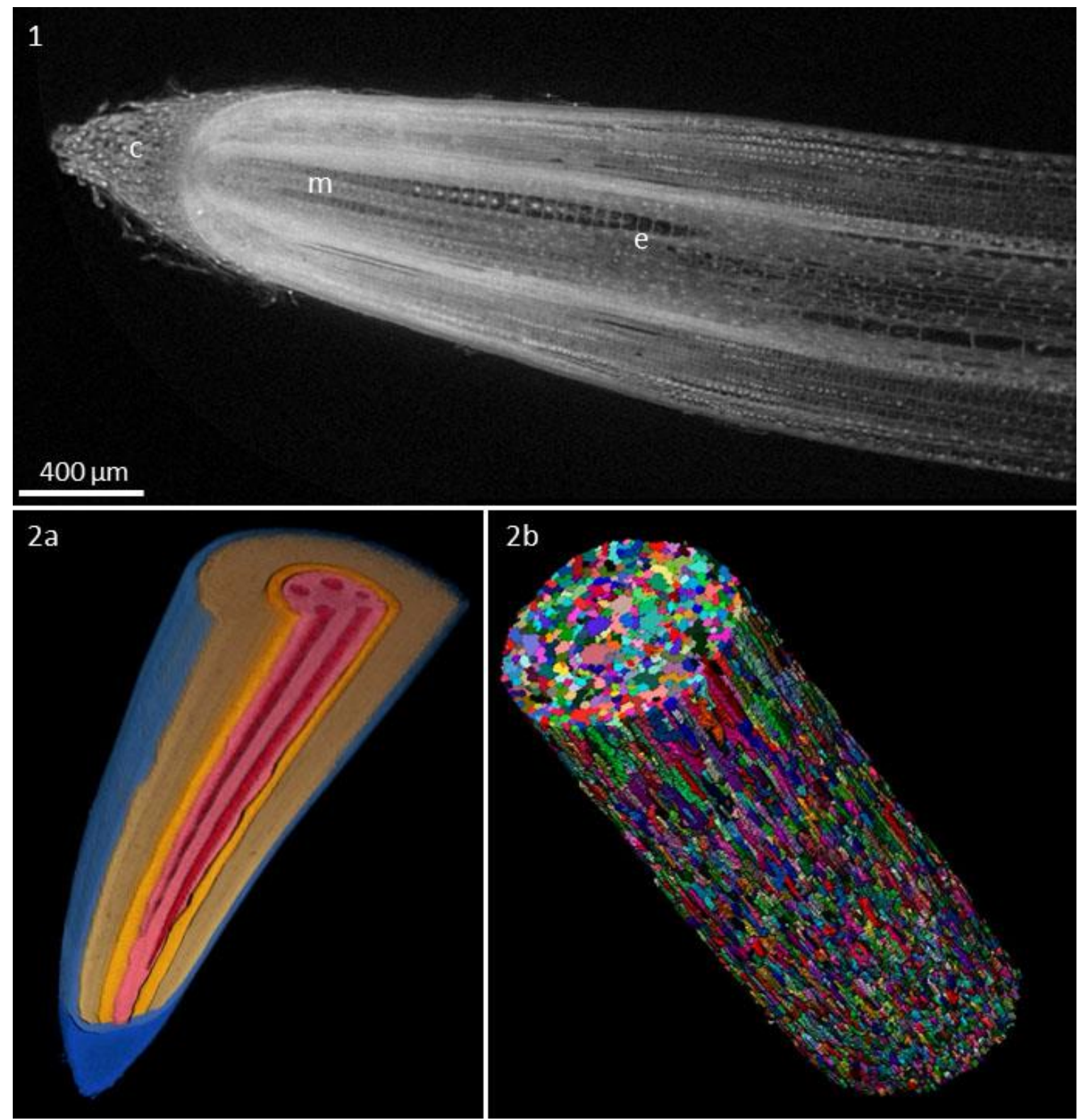

Figure 1. 1. Maize root cap (c), meristematic (m) and elongation (e) zones, contrasted with osmium tetroxide-uranyl acetate-lead citrate and scanned by XRM at $2 \mu \mathrm{m}$ voxel resolution. 2a. Computational segmentation of maize root into tissues (a) and individual cells (b).

\section{References}

[1] CN Topp et al, J Integrative Plant Biol 58 (2016), p. 213.

[2] AL Bray and CN Topp, Plant Cell Physiol 59 (2018), p. 1919

[2] N Jiang et al, Plant Cell 31 (2019), p. 1708.

[3] A Gamisch et al, PLoS One 8 (2013), p. e72688.

[4] A Bellaire et al, New Phytol 202 (2014), p. 322.

[5] YM Staedler et al, J Exper Bot 69 (2018), p. 525.

[6] J Jeiter et al, Int J Plant Sci 179 (2018), p.275.

[7] This work is supported by collaborative agreements with Valent BioSciences, LLC, and Sumitomo Chemical Company. 